\title{
Comparison of an enzyme immuno assay (Antigenz Chlamydia) with cell culture for the detection of genital chlamydial infection in high and low risk populations
}

\author{
G Mumtaz, S Clark, GL Ridgway, CJ Miller, B Johal, E Allason Jones
}

\begin{abstract}
Objective-To evaluate a new enzyme immuno assay, Antigenz Chlamydia, for the detection of chlamydial antigen in urogenital samples using cell culture as the gold standard for comparison and direct immuno fluorescence to aid resolving the discrepancies.

Subjects-212 men and 303 women attending an STD clinic and 404 women of child bearing age attending gynaecological clinics.

Results-Sensitivity, specificity, predictive value of positive result and predictive value of negative result of the new test were $77.6 \%, 99.0 \%, 94 \cdot 3 \%$, and $95 \cdot 7 \%$ respectively in the high risk population with a prevalence of $16.5 \%$. In the low risk population the figures were $84 \cdot 2 \%$, $94.5 \%, 43 \cdot 2 \%$ and $99.2 \%$ respectively with a prevalence of $4 \cdot 7 \%$.

Conclusion-Antigenz Chlamydia is an easy and quick test to perform. The test is suitable for use in high risk and low risk populations provided all positive samples are confirmed.
\end{abstract}

(Genitourin Med 1993;69:119-122)

\section{Introduction}

Chlamydia trachomatis is recognised as one of the most prevalent sexually transmitted pathogen. Although genital infection with this organism is often low grade and chronic, it remains an important cause of PID and its sequelae.

Several non-cultural antigen detection techniques are now available for the diagnosis of genital chlamydial infection. ${ }^{1}$ Evaluation in a high risk population is usually statistically favourable towards the new test with regard to the prediction value of a positive result, whereas the converse may well be the case in a low risk population. Only a few of the tests currently available incorporate a confirmatory test, which will reduce the possibility of false positive results. ${ }^{2}$

We describe here the evaluation of a new enzyme immunoassay (EIA)(Northumbria Biologicals Ltd, UK) in high and low risk populations against a standard cell culture technique. Discrepant results were further analysed using direct immunofluorescence (DIF). A confirmatory test (blocking assay) was available for a proportion of the samples.

\section{Materials and methods}

Patients

The high risk group comprised 212 men and 303 women attending the Genitourinary Medicine Department at University College Hospital (UCH) for investigation of nongonococcal infection. The low risk group comprised 404 women of childbearing age attending gynaecological clinics (colposcopy, gynaecological OPD, termination of pregnancy, family planning) at UCH for reasons other than sexually transmitted disease. Patients who had received antimicrobial chemotherapy during the previous 4 weeks were excluded.

\section{Specimens}

From women, two cervical specimens were obtained after wiping the cervix. From men, two urethral swabs were taken by insertion of an aluminium mounted swab $4 \mathrm{~cm}$ into the urethra. One swab was placed in EIA transport medium supplied by the kit's manufacturer (Northumbria Biologicals Ltd) and the other into 2SP chlamydial transport media. The order of collection was determined by reference to a random number table. ${ }^{3}$

\section{Chlamydia culture}

Cycloheximide treated McCoy cells were used to grow Chlamydia trachomatis. ${ }^{4}$ Two cell culture tubes were inoculated per sample. The coverslip monolayer from one tube was stained with Lugol's iodine and examined after 48-72 $\mathrm{h}$ incubation for the presence of inclusions. The contents of the 2 nd tube were passaged on to a fresh cell sheet and examined using an immunofluorescent culture confirmation test (Syva Company, Palo Alto C A) after incubation for a further $48-72 \mathrm{~h}$.

\section{Enzyme immunoassay}

Samples collected into $1 \mathrm{ml}$ of the EIA storage transport medium were stored at $4^{\circ}-8^{\circ} \mathrm{C}$ for up to 8 days before testing according to the manufacturer's instructions. Samples were vortex mixed for 15 seconds and then $200 \mu \mathrm{l}$ was withdrawn and kept at $-70^{\circ} \mathrm{C}$ in Eppendorf vials for DIF.

The remainder of the sample along with the kit's positive and negative controls were boiled for 10 minutes and cooled at room temperature prior to being vortexed for a further 15 seconds. Sufficient numbers of pretreated strips of microtitration wells were selected to account for the number of samples plus two negative and one positive controls. 
Sheep anti murine IgG (25 $\mu \mathrm{l})$ conjugated to horse radish peroxidase was added to all designated wells first, followed by $200 \mu \mathrm{l}$ of sample. Antibody (murine monoclonal antibody against chlamydial LPS) $50 \mu \mathrm{l}$ was then added to this mixture, the strips were covered with sealing film, gently tapped to $\mathrm{mix}$ the contents thoroughly, and then incubated for 1 hour at $37^{\circ} \mathrm{C}$. Using an automatic washer (Denley well wash 4 ), the strips were vigorously washed with the rinse solution provided. Freshly made chromogenic substrate (tetramethyl benzidine (TMB), $200 \mu \mathrm{l}$ was added to each well. The wells were sealed and incubated at room temperature for 20 minutes. Stopping solution $\left(2 \mathrm{~N} \mathrm{H}_{2} \mathrm{SO}_{4}\right) 50 \mu \mathrm{l}$ was added and the contents mixed before reading the absorbance at $450 \mathrm{~nm}$ in a Titertech Multiscan plate reader. The cut off point was taken as the average absorbance of the negative controls plus $0 \cdot 1$ absorbance unit. Samples giving readings below this value were regarded as negative. Samples giving readings between the cut off value and cut off value plus 0.05 absorbance unit were considered to be in the grey zone. Samples giving values greater than the upper limit of the grey zone were scored as positive. All samples which gave readings in the grey zone or did not agree with the culture results were retested for reproducability.

\section{Direct immunofluorescence}

The $200 \mu \mathrm{l}$ of the EIA sample which was stored at $-70^{\circ} \mathrm{C}$ was thawed out and spun in a MSC Microcentaur at $13000 \mathrm{rpm}$ for 20 minutes. The supernatant fluid was removed and the pellet resuspended in $180 \mu \mathrm{l}$ of PBS, vortexed hard and spun again. The supernatant fluid was removed and the pellet resuspended in $100 \mu \mathrm{l}$ of PBS, vortexed hard and spotted on to the wells of a teflon-coated slide and air dried. This preparation was fixed with methanol and stained with an immunofluorescent anti Chlamydia trachomatis MOMP monoclonal antibody (Syva Microtrak) according to the manufacturer's instructions. Specimens were examined by immunofluorescence microscopy for typical chlamydial elementary bodies. Samples containing one or more disc shaped, uniformly stained, fluorescing green bodies were regarded as positive.

\section{Blocking assay}

Samples giving a positive EIA chlamydial result were re-assayed in duplicate. For this test, only one modification was made to the original assay procedure. A solution containing rabbit anti-chlamydial serum (blocking antibody) was added to one microwell, and a solution containing an equivalent concentration of normal rabbit serum (control) was added to a second microwell after addition of the conjugate and the sample and prior to the addition of the anti LPS monoclonal antibody. The incubation period and the washing step and colour development were followed as previously. The blocking serum competes for antigenic site with the anti LPS mono- clonal antibody. This results in a lowering of the test absorbance. A $50 \%$ or greater reduction in net absorbance of the blocked assay, compared with the net absorbance of the unblocked assay, confirms the presence of chlamydiae in the sample. Active chlamydial infection was defined as growth in cell culture, or DIF positive.

\section{Results}

\section{High risk population of men}

By the use of cell culture with passage, the prevalence of Chlamydia trachomatis in this high risk population of men was found to be $16.0 \%(34 / 212)$. There were 26 co-positives (true positive), 166 co-negatives (true negative), 8 culture positive-EIA negative (false negative), 7 culture negative EIA positive (false positive) and 5 EIA grey zone culture negative samples. None of the 8 false negative samples were DIF positive, suggesting low or absent antigen. The 5 grey zone samples, all retested as EIA negative, but 2 were DIF positive. Therefore, $2 / 5$ are recorded as false negative, and $3 / 5$ as true negative. Three of the 7 false positives were negative on repeat and DIF negative, and are recorded as true negative. Three more samples out of this group were DIF positive and are recorded as true positive. Therefore the overall prevalence (culture or DIF positive) in this group of patients was $18.3 \%(39 / 212)$. Of the 212 patients in this group 91 had NGU (43\%). C. trachomatis was confirmed in 24/91 (26.4\%); of these patients Neisseria gonorrhoeae was isolated in $5 \cdot 1 \%$ ( 11 out of 212 patients). Three $(27 \cdot 3 \%)$ of these patients yielded $C$ trachomatis on culture.

The sensitivity, specificity, predictive value of positive results (PVP) and predictive value of a negative result (PVN) in this group of patients were found to be $74.4 \%, 99 \cdot 4 \%$, $96.7 \%$ and $94.5 \%$ respectively with the agreement between the two tests (EIA v cell culture) being $90 \cdot 6 \%$. (table 1 )

\section{High risk population of women}

By the use of the cell culture with passage, the prevalence of Chlamydia trachomatis in this high risk population of women was found to be $14 \cdot 8 \%(45 / 303)$. There were $36 \mathrm{co}-$ positive, 247 co-negative, 9 culture positiveEIA negative (false negative), 6 culture negative EIA positive (false positive) and 5 EIA "grey zone" culture negative samples. All

Table 1 Analysis of EIA $v$ cell culture results with DIF on discrepant results in high risk men

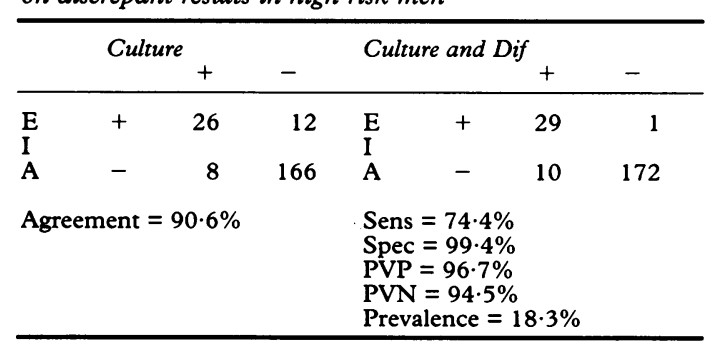


Table 2 Analysis of EIA $v$ cell culture results with DIF on discrepant results for high risk group of women

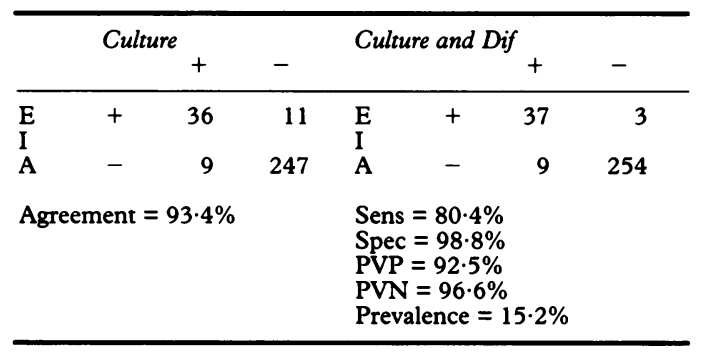

Table 3 Combined analysis of ELA $v$ Culture results with DIF on discrepant samples for high risk group of patients

\begin{tabular}{lccc}
\hline Culture ED Dif & & + & - \\
\hline E & + & 66 & 4 \\
I & - & 19 & 426 \\
A & & & \\
Sens $=77 \cdot 6 \%$ & & \\
Spec $=99 \cdot 0 \%$ & & \\
PVP $=94 \cdot 3 \%$ & & & \\
PVN $=95 \cdot 7 \%$ & & \\
Prevalence $=16.5 \%$ & &
\end{tabular}

five samples which were in the "grey zone" retested as negative and were DIF negative. These were recorded as true negatives. Out of six false positive samples one was DIF positive and recorded as true positive and two were DIF negative and retested as negative and were recorded as true negative. None of the nine culture positive-EIA negative samples were DIF positive suggesting low or absent antigen. Therefore the overall prevalence (culture or DIF positive) in this group of patients was $15 \cdot 2 \%(46 / 303)$.

The sensitivity, specificity, PVP and PVN in these patients were found to be $80.4 \%$, $98 \cdot 8 \%, 92 \cdot 5 \%$ and $96.6 \%$ respectively with agreement between the two tests (EIA v culture) being $93 \cdot 4 \%$. (table 2 ).

The combined results for both high risk groups are shown in Table 3. The combined sensitivity, specificity, PVP and PVN were $77.6 \%, 99.1 \%, \quad 94.3 \%$ and $95.7 \%$ respectively.

\section{3 low risk population of women}

By the use of cell culture with passage, the prevalence of Chlamydia trachomatis in this low risk group of women was found to be $3 \cdot 7 \%(15 / 404)$. There were 12 co-positive, 360 co-negatives, 21 EIA positive-culture negative, 8 EIA "grey zone" - culture negative and 3 EIA negative-culture positive samples. Four out of 8 samples which were in the "grey zone" retested as EIA negative and were DIF negative. These were scored as true negative. The remaining four retested as "grey zone" or positive but were DIF negative. These were scored as false positive. Only four out of the 21 culture negative-EIA positive samples were positive by DIF test. These were scored as true positive. Therefore the overall prevalence (culture or DIF) was $4.7 \%$ (19/404) (table 4). Only one out of three false negative samples were DIF positive.

The results obtained here show a sensitivity, specificity, PVP and PVN of $84 \cdot 2 \%$,
Table 4 Analysis of ELA $v$ cell culture results with DIF on discrepant results for low risk women

\begin{tabular}{|c|c|c|c|c|c|c|c|}
\hline & $\mathrm{Cu}$ & & & $\mathrm{Cu}$ & & & \\
\hline & & + & - & & & + & - \\
\hline$\underset{I}{E}$ & + & 12 & 29 & $\begin{array}{l}\mathrm{E} \\
\mathrm{I}\end{array}$ & + & 16 & 21 \\
\hline A & - & 3 & 360 & A & - & 3 & 364 \\
\hline & nent & $2 \cdot 1$ & & $\begin{array}{l}\text { Ser } \\
\text { Sp } \\
\text { PV } \\
\text { PV } \\
\text { Pre }\end{array}$ & 9 & & \\
\hline
\end{tabular}

$94.5 \%, 43.2 \%$ and $99.2 \%$ respectively with the agreement between the EIA and cell culture being $92 \cdot 0 \%$.

Towards the end of this study, a blocking antibody became available. Only those samples which had sufficient material left to do the confirmatory test were available for the blocking assay. These included all the 12 copositive and 11 of 24 EIA positive-culture negative samples. All the co-positive samples were confirmed as positive by the blocking test. Four of the remaining 11 showed no drop in the O.D. with the blocking antibody, and the other seven gave a negative signal in both control and blocking assay wells. Only one of the three samples which was culture negative-EIA positive-DIF positive was available for reassay. This sample was not confirmed as positive using the blocking test.

\section{Discussion}

Over the years several rapid antigen detection techniques for chlamydiae have become available and are now widely used. EIAs have the advantage of being objective and easy to automate. The remaining material may be used for confirmation of the positive results either using a DIF test ${ }^{5}$ or by a confirmatory blocking assay. ${ }^{2}$ However, in different hands, EIAs show different sensitivities and specificities with values ranging from $62-98 \%$ and 92 $100 \%$ respectively. ${ }^{6}$

We employed cycloheximide-treated McCoy cells, with passage of cultures stained by immunofluorescent monoclonal antibody (Syva culture confirmation) for this evaluation. In addition, all discrepant results were studied further by performing a DIF test on the EIA buffer.

A number of variables may affect the outcome of a comparison between EIA and tissue culture in a trial situation eg quality of samples, transportation and tissue culture sensitivity. These variables cause difficulties when trials performed at different times are compared. However, bearing in mind these constraints, in the high risk population group, we found the overall sensitivity $(77 \cdot 6 \%)$ was low compared with our results for the Abbott Chlamydiazyme $^{3}$ or Pharmacia EIA $^{7}$ tests, $(92.5 \%$ and $88 \%$ respectively), but of the same order as our findings with the Ortho test $(78 \cdot 2 \%)^{8}$ and Unipath Clearview $(79 \cdot 4 \%){ }^{9}$ The specificity was $99 \cdot 1 \%$ compared with 97\% an:. 99\% fe: Abbott and Pharmacia tests and $95.8 \%$ and $100 \%$ for Ortho and 
Clearview respectively. The predictive value of a positive test was also comparable with Abbott and Pharmacia tests (94.3\% compared with $94.6 \%$ and $96 \%$ respectively). It should be noted that sensitivity and predictive value of a positive test is affected by the incidence of infection which was lower in this study $(16.5 \%$ compared with $27 \cdot 1 \%$ in the Abbott study and $22.3 \%$ in the Pharmacia study). As in all commercial EIA tests, the predictive value of a negative test was high $(95 \cdot 7 \%)$.

There was a total of 40 discrepant results. None of the 17 culture positive, EIA negative samples were DIF positive suggesting low or absent antigen. Twenty-three samples were culture negative, EIA positive. Four of 23 were DIF positive and recorded as true positive. Two of 23 "grey zone" results retested as negative but were DIF positive. These have been recorded as false negatives. Although this finding underscores the importance of repeating positive EIA results, irrespective of whether a confirmatory test is available and used it must not undermine the value of further investigating "grey zone" results. Thirteen of 23 were negative on retest and recorded as true negative. Thus, combining culture and DIF and retesting EIA resolved $17 / 40$ discrepant results. Further resolution of the discrepant results was not possible as the blocking assay was not available to us at the time.

In the low risk population of women, we detected four EIA positive, DIF positive, culture "misses" out of the total of 21 specimens which gave a positive signal initially. Two out of three false negative samples yielded $C$ trachomatis only after cell culture passage. One of these two samples was DIF negative. The third sample was also DIF negative suggesting a low antigenic load. With the exception of the PVP value $(43.2 \%)$, these results are comparable with our work with high risk groups. The number of false positive results and samples giving signals in the "grey zone" area in this study is quite high. However, we were aware that during this part of the study our automatic washer failed to function properly. Some of available samples on repeat were found to be negative when hand washed or the corrected washer was used. Later in the study when a confirmatory test became available (blocking antibody), we were able to perform this test on a proportion of the EIA positive-culture negative and all of the copositive samples. The washing step in the blocking assay was performed by hand. All but one of the results were in agreement with culture or DIF. As we do not have blocking antibody data on all the initial EIA positive signals, we are unable to incorporate them in the final calculations. However, this problem with washing has further emphasised the need for a confirmatory test for all the EIAs available for chlamydiae. The use of a confirmatory test helps the interpretation of the positive signals which may have risen as the result of bad washing, cross reaction with excess numbers of other microorganisms, splash or clerical errors. By the virtue of not requiring yet another technique, an inbuilt confirmatory test is also more convenient to use.

We found NBL Antigenz Chlamydia a rapid and straightforward test. Our limited experience with the blocking assay suggests that the test benefits from the additional blocking assay. When used, all positive results must be repeated and a confirmatory test performed.

1 Ridgway GL, Taylor-Robinson D. Current problems in microbiology: 1 Chlamydial infections: Which laboratory test? 7 Clin Pathol 1991;44:1-5.

2 Moncada J, Schachter J, Bolan G, et al. Confirmatory assay increases specificity of chlamdiazyme test for Chlamydia trachomatis infection of the Cervix. $\exists$ Clin Microbiol 1990;28:1770-3.

3 Mumtaz G, Mellars BJ, Ridgway GL, Oriel JD. Enzyme immunoassay for the detection of Chlamydia trachomatis antigen in urethral and endocervical swabs. $\mathcal{F}$ Clin Pathol 1985;38:740-2.

4 Ripa KT, Mardh PA. Cultivation of $C$. trachomatis in cycloheximide treated McCoy cells. $f$ Clin Microbiol 1977;6:328-33.

5 Ridgway GL, Oriel JD, Mumtaz G, Mellars B. Comparison of methods for detecting Chlamydia trachomatis. F Clin Pathol 1985;39:232-3.

6 Taylor-Robinson D, Thomas BJ. Laboratory techniques for the diagnosis of chlamydial infection. Genitourin Med 1991;67:256-66.

7 Mumtaz G, Ridgway GL, Nayagam AT, Oriel JD. Enzyme immunoassay compared with cell culture and immunofluorescence for detecting genital chlamydia. $\mathcal{J}$ Clin Pathol 1989;42:658-60.

8 Mumtaz G; Ridgway GL, Nayagam AT, Robinson AJ. Comparison of an enzyme immunoassay (Ortho) with cell culture and immunofluorescence for the detection of genital chlamydial infection. International fournal of STD E AIDS 1990;1:187-90.

9 Ridgway GL, Mumtaz G, Allason-Jones E, Bingham JB. Solid phase immunoassay for Chlamydia trachomatis. Genitourin Med 1991;67:268-71. 